\title{
Changes of the Unique Odontogenic Properties of Rat Apical Bud Cells under the Developing Apical Complex Microenvironment
}

\author{
Jun Fang ${ }^{1,2, a}$, Liang Tang ${ }^{2,3, a}$, Xiao-hui Liu ${ }^{1,2}$, Ling-ying Wen ${ }^{1 *}$, Yan Jin $^{2,3 *}$ \\ ${ }^{1}$ Department of Pedodontics, School of Stomatology, Fourth Military Medical University, Xi'an, China \\ ${ }^{2}$ Department of Oral Histology and Pathology, School of Stomatology, Fourth Military Medical University, Xi'an, China \\ ${ }^{3}$ Research and Development Center for Tissue Engineering, Fourth Military Medical University, Xi'an, China
}

\begin{abstract}
Jun Fang, Liang Tang, Xiao-hui Liu, Ling-ying Wen, Yan Jin. Changes of the Unique Odontogenic Properties of Rat Apical Bud Cells under the Developing Apical Complex Microenvironment. International Journal of Oral Science, 1(1): 26-33, 2009
\end{abstract}

Aim To characterize the odontogenic capability of apical bud and phenotypical change of apical bud cells (ABCs) in different microenvironment.

Methodology Incisor apical bud tissues from neonatal SD rat were dissected and transplanted into the renal capsules to determine their odontogenic capability. Meanwhile $\mathrm{ABCs}$ were cultured and purified by repeated differential trypsinization. Then ABCs were cultured with conditioned medium from developing apical complex cells
CLC number: Q813.1

\section{Introduction}

Tooth development is characterised by sequential and reciprocal interactions between the odontogenic epithelium and neural crest derived mesenchyme as well as other ectodermal organs, resulting in balanced growth including proliferation and apoptosis, determination and differentiation of cell populations forming the tooth germ followed by a deposition of tooth-specific calcified matrix (Thesleff et al., 1995; Jernvall et al., 2000; Pispa et al., 2003; Tucker et al., 2004). In most mammalian developing molars, cervical loop derived Hertwig's epithelial root sheath (HERS) induces and conducts tooth root and periodontal tissue development after tooth crown formation (Hammar-
(DAC-CM). Immunocytochemistry, reverse transcriptase polymerase chain reaction (RT-PCR) and scanning electron microscope (SEM) were performed to compare the biological change of ABC treated with or without DAC-CM.

Results First we confirmed the ability of apical bud to form crown-like structure ectopically. Equally important, by using the developing apical complex (DAC) conditioned medium, we found the microenvironment created by root could abrogate the "crown" features of ABCs and promote their proliferation and differentiation.

Conclusion $\mathrm{ABCs}$ possess odontogenic capability to form crown-like tissues and this property can be affected by root-produced microenvironment.

Keywords apical bud, tooth root development, differentiation ström et al., 1996; Luan, et al., 2006; ZeichnerDavid et al., 2003). In contrast, continuous growth throughout the lifetime can be observed in the murine incisor, rendering it a special tooth type (Smith et al., 1975; Harada et al., 1999; Harada et al., 2002; Kawano et al., 2004; Harada et al., 2004; Ohshima et al., 2005). The explanation for this speciality lies in the fact that the specific epithelial structure, i.e. apical bud, is composed of a large amount of stellate reticulum and basal epithelium so the apical bud possesses the capacity for constant production of ameloblasts and thus disturbs the transition from crown to root. Equally important, the apical bud are proved to be endowed with enriched adult dental epithelial stem cells exerting the above mentioned function. 
Fang et al. Changes of Odontogenic Properties of Rat Apical Bud Cells under Microenvironment

Several studies have reflected the putatively unique feature and function of apical bud cells (ABCs) in vitro. Through interaction with mesenchymal cells, ABCs can differentiate along ameloblast cell lineage (Morotomi et al., 2005). Our previous study demonstrated that the inductive microenvironment generated by $\mathrm{ABCs}$ could promote the lineage-specific differentiation of undifferentiated dental pulp stem cells and even skew the differentiation of non-odontogenic cells such as bone marrow stromal stem cells and follicle dermal papilla mesenchymal cells towards odontogenic cells (Yu et al., 2007; Wu et al., 2008). ABCs combined with dental pulp stem cells in the form of cell pellets resulted in distinct tooth crown structure morphogenesis ectopically renal capsule (Yu et al., 2007). However, unresolved issues remain including the characterization of $\mathrm{ABCs}$ in defined culture condition, and whether the continuous ameloblast-producing ability could be affected by a microenvironment created by root/ periodontal tissues represented by a developing apical complex (DAC) (Yang et al., 2008; Xu et al., 2008). In the present study, we performed experiments to provide answers for these issues. The results represent an important step toward further understanding of biological functions of ABCs.

\section{Methods and materials}

\section{apical bud cells isolation and culture}

Sprague-Dawley rats of 5 days postnatal (dpn) were purchased from the Laboratory Animal Research Centre of Fourth Military Medical University. ABCs were isolated and cultured as described by our previous research (Yu et al., 2007). After decase under anaesthesia, the lower incisors were carefully extracted from 5-day postnatal (dpn) SD rats. The enamel organ and dental papilla were physically separated from each other with forceps (Figure 1C and 1D), and the apical buds were carefully isolated with injection needles. ABCs were enzymically isolated from apical bud tissues, and single-cell suspensions were generated by filtration through a $70 \mu \mathrm{m}$ strainer, placed into 6-well plates at $1 \times 10^{4}$ cells/ well in DMEM (Dul- becco's modified Eagle's medium) /F12 (Gibco BRL, Grand Island, USA) supplemented with $10 \%$ fetal bovine serum (FBS; Gibco-BRL, USA) until full confluence. Through repeated differential trypsinization as reported in Jinga et al. (2000), purified epithelial-like cells were obtained excluding the contamination of fibroblastic cells. Epithelial-like ABCs were cultured in keratinocyte serum-free medium (KSFM; GIBCO-BRL, USA) supplemented with $0.015 \mathrm{ng} / \mathrm{mL}$ epidermal growth factor (EGF; Invitrogen Corp., USA) and $25 \mu \mathrm{g} / \mathrm{mL}$ bovine pituitary extract (BPE; Invitrogen Corp., USA).

\section{Subrenal transplantation and analysis of apical bud tissue}

Our animal use protocols (rat) were reviewed and approved by the Animal Care Committee of Fourth Military Medical University. Apical bud tissues were dissected from the end of $5 \mathrm{dpn}$ SD rat incisor, then seeded directly into the renal capsules with modified pipette tips on the left side of rat host. The transplantation process was identical as previously described by our team (Yu et al., 2006a; Yu et al., 2006b). In total, we implanted 20 apical bud tissues into renal capsules. The development of these implants was assessed after 14-day incubation. Retrieved explants were processed in the serial paraffin sections and analyzed by Haematoxylin \& Eosin (HE) staining.

\section{Preparation of conditioned medium from deve- loping apical complex cells}

40 intact first molar tooth germs of postnatal $10 \mathrm{dpn}$ SD rats were isolated. DAC was isolated from the border of crown and root of the molar with an injection needle along the margin of hard tissue of crown. Then the separated tissues were minced into small pieces $(<0.1 \mathrm{~cm})$ and digested with mixture of Dispase I $(0.3 \mathrm{U} / \mathrm{mL}$; Gibco-BRL, USA) and type I collagenase $(2.5 \mathrm{mg} / \mathrm{mL}$; GibcoBRL, USA) for 1 hour at $37^{\circ} \mathrm{C}$. Single cell suspension was filtered by passing through $70 \mu \mathrm{m}$ cell stainer. Cells were suspended in DMEM supplemented with $10 \%$ FBS and plated into $25 \mathrm{~cm}^{2}$ culture flasks at a density of $1 \times 10^{5}$ cells $/ \mathrm{mL}$, with the medium changed every other day, and the cells 
cultured under $5 \% \mathrm{CO}_{2}$ at $37^{\circ} \mathrm{C}$. Three days after the last change of medium, the media were collected and centrifuged at $3000 \mathrm{r} / \mathrm{min}$ for 15 minutes. The supernatants, which were mixed with an equal volume of fresh DMEM supplemented with $10 \%$ FBS, were then used as APMG-CM and stored at $-20^{\circ} \mathrm{C}$.

\section{Immunocytochemistry analysis}

The $\mathrm{ABCs}$ in both groups were seeded on to coverslips at a density of $2 \times 10^{5}$ cells $/ \mathrm{mL}$, subcultured for 2 days and then fixed with $4 \%$ polyoxymethylene. Immunocytochemical analyses were performed with the streptavidin-biotin complex method according to the manufacturer's recommended protocol. Antibodies included: mouse antivimentin (1:30; DAKO Diagnostics, USA), mouse anti-cytokeratin-14 (1:100; Chemicon, USA), rabbit anti-amelogenin (1:100; Santa Cruz Biotechnology, USA). The specimens were counterstained with haematoxylin, and all the samples were examined under an Olympus compound microscope (Olympus Optical Co. Ltd., Japan).

\section{Reverse transcriptase polymerase chain reac- tion}

APMG-CM treated and untreated ABCs at 90\% confluence were harvested. Total RNA was isolated by means of TRIzol ${ }^{\circledR}$ reagent (Invitrogen Corp., USA) and was reversetranscribed using the Super-Script ${ }^{\mathrm{TM}}$ First-Strand Synthesis System (Invitrogen Corp., USA) for reverse transcriptase polymerase chain reaction (RT-PCR) followed the manufacturer's instructions. Primer sequences were as follows:

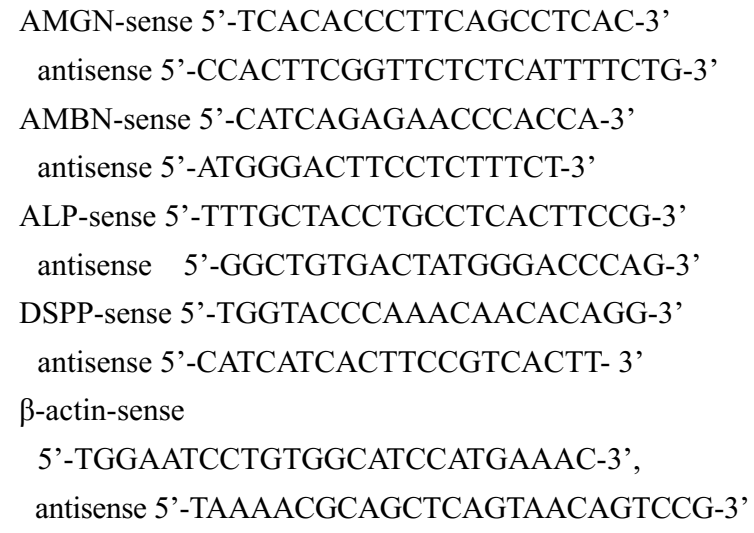

After 30 cycles, the PCR products were separated by using $2 \%$ agarosegel electrophoresis and were stained with ethidium bromide, with digital images recorded on a UV background. This experiment was repeated three times to confirm the results.

\section{Scanning electron microscope of apical bud cells}

The $\mathrm{ABCs}$ were seeded on to coverslips at a density of $2 \times 10^{5}$ cells $/ \mathrm{mL}$ for additional culture and then fixed with $4 \%$ polyoxymethylene. The cells were dehydrated in a series of ethanol and embedded in Epon 812 resin (Taab Laboratories Equipment Ltd., UK). The samples were viewed with scanning electron microscope (SEM; HITA CHI S-2700, Hitachi Corp., Japan) after coating the samples with a thin layer of gold under vacuum.

\section{Results}

\section{Characterization of apical bud cells in estab- lished culture system}

The apical bud was observed as a protrusion of soft tissue located at the end of rat incisor. We established procedures for getting relative purified dental epithelial cells from apical bud (Yu et al., 2007). In the 3rd day of primary culture, attached cells exhibited heterogeneous appearance containing both cobblestone-like epithelial cells and polygonal or spindle-shaped mesenchymal cells (Figure 1B). Cells in fibroblast appearance entirely disappeared in purified ABCs (Figure 1C). Remnant $\mathrm{ABCs}$ continued to grow and reached confluence under keratinocyte serum-free medium (K-SFM). To assess the epithelial and odotogenic characteristics of $\mathrm{ABCs}$, we performed immunostaining against cytokeratin (CK)-14 (Figure 1D) and amelogenin (AMGN, Figure 1E). Almost all of the ABCs positively expressed CK-14 and AMBN, while none stained positive to vimentin (Figure $1 \mathrm{~F})$, which was deemed as marker for mesenchymal cell. RT-PCR analysis revealed that ABCs expressed transcripts AMGN and AMBN which were representative of ameloblasts (Figure 1G). ALP expression was seen in cultured ABCs, which 
meant dental epithelial cells from apical bud gained features of maturation during culture (Wise, et al., 1989). When the apical bud was dissected from the end of incisor and then implanted into renal capsule of a syngeneic rat for 2 weeks, mineralized tissue formed and was further proved by HE staining to be a crown-like tissue (Figure $2 \mathrm{~A}, 2 \mathrm{~B}, 2 \mathrm{C}$ ), in which distinct formation of ameloblast, enamel, dentin and odontoblast was observed. These results well argued that ectopically implanted apical bud tissue retains the ability to form crownlike tissue.

\section{Conditioned medium from developing apical complex promotes the differentiation of apical}

\section{bud cells}

DAC was isolated from the border of crown and root of $10 \mathrm{dpn}$ SD rat first mandibular molar as described previously (Yang et al., 2008; Xu et al., 2008) (Figure 3A). Cells isolated from the DAC grown in DMEM/F12 medium with $10 \%$ FBS contained a mixture of cells with both an epithelial, cobblestone-shaped phenotype, and a fibroblast spindle shape (Figure 3B). The DAC conditioned medium was bright and transparent and free of cell fragments contamination.

To determine whether ABCs go with the similar biological events treated with DAC-CM in vitro, we observed the morphological changes and expression pattern of ABCs. During the inductive cul-
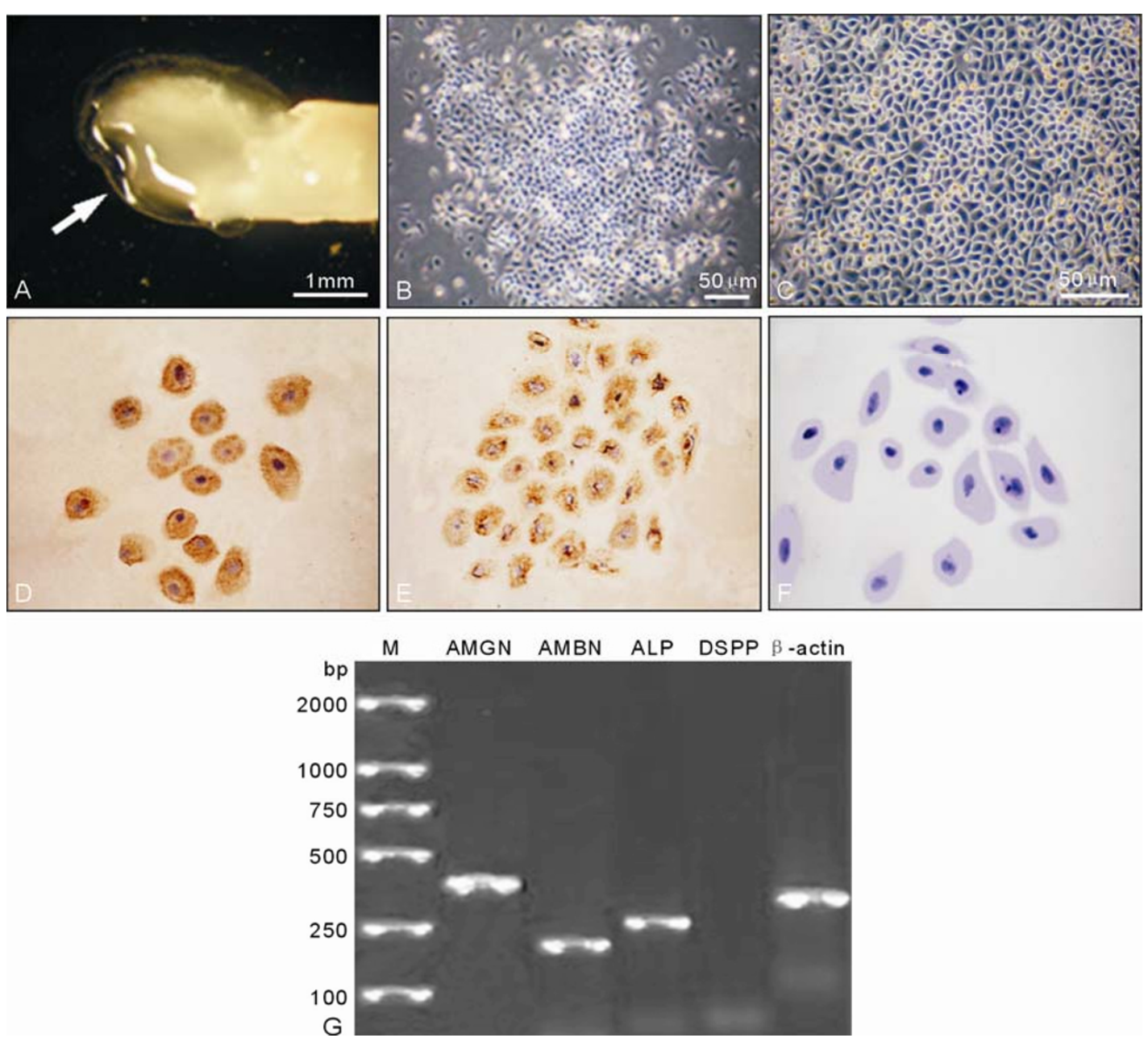

Figure 1 Isolation and characterization of $\mathrm{ABCs}$

(A): The macroscopic view of apical bud as the arrow indicates. (B): In primary culture cells exhibited heterogeneous appearance containing both cobblestone-like epithelial cells and polygonal or spindle-shaped mesenchymal cells. (C): Only cells in epithelial-like appearance existed in purified ABCs. (D): ABCs were positively immunostained for CK-14. (E) :AMGN was characteristically expressed in ABCs. (F): ABCs were negatively stained for vimentin. (G): RT-PCR analysis revealed that ABCs expressed transcripts of AMGN, AMBN and ALP but not DSPP. Scale bar is shown as indicated. 

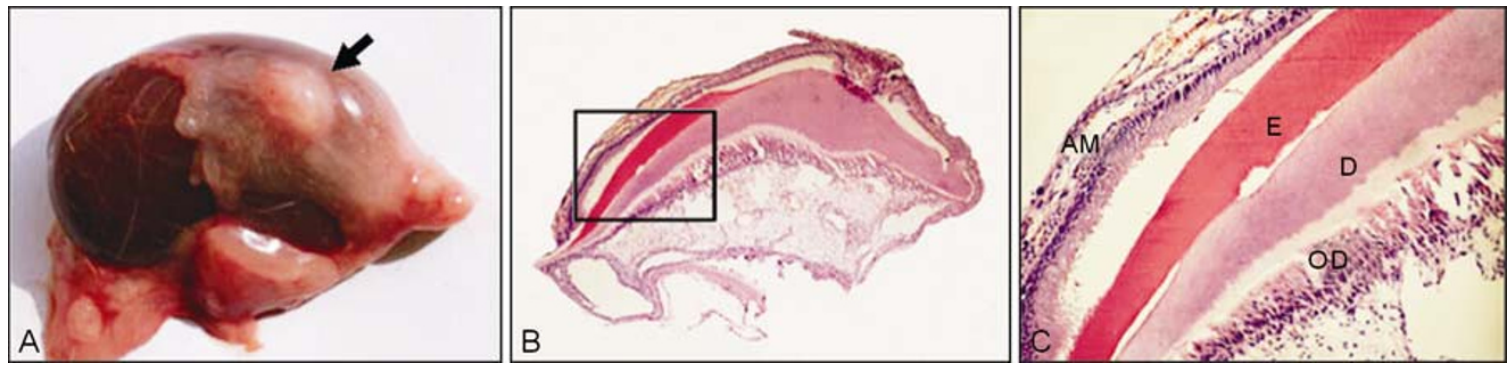

Figure 2 Formation of tooth crown like structure by apical bud tissues implanted in rat renal capsule

(A): Macroscopic view of explant of apical bud tissues (indicated by arrow) after 2-week subrenal implantation. (B): Low power microscopic view of HE staining of representative explant. (C): Higher magnifications of the boxed area in B, which shows distinct formation of enamel (E) secreted by ameloblast (AM), and dentin (D) produced by odontoblast (OD).
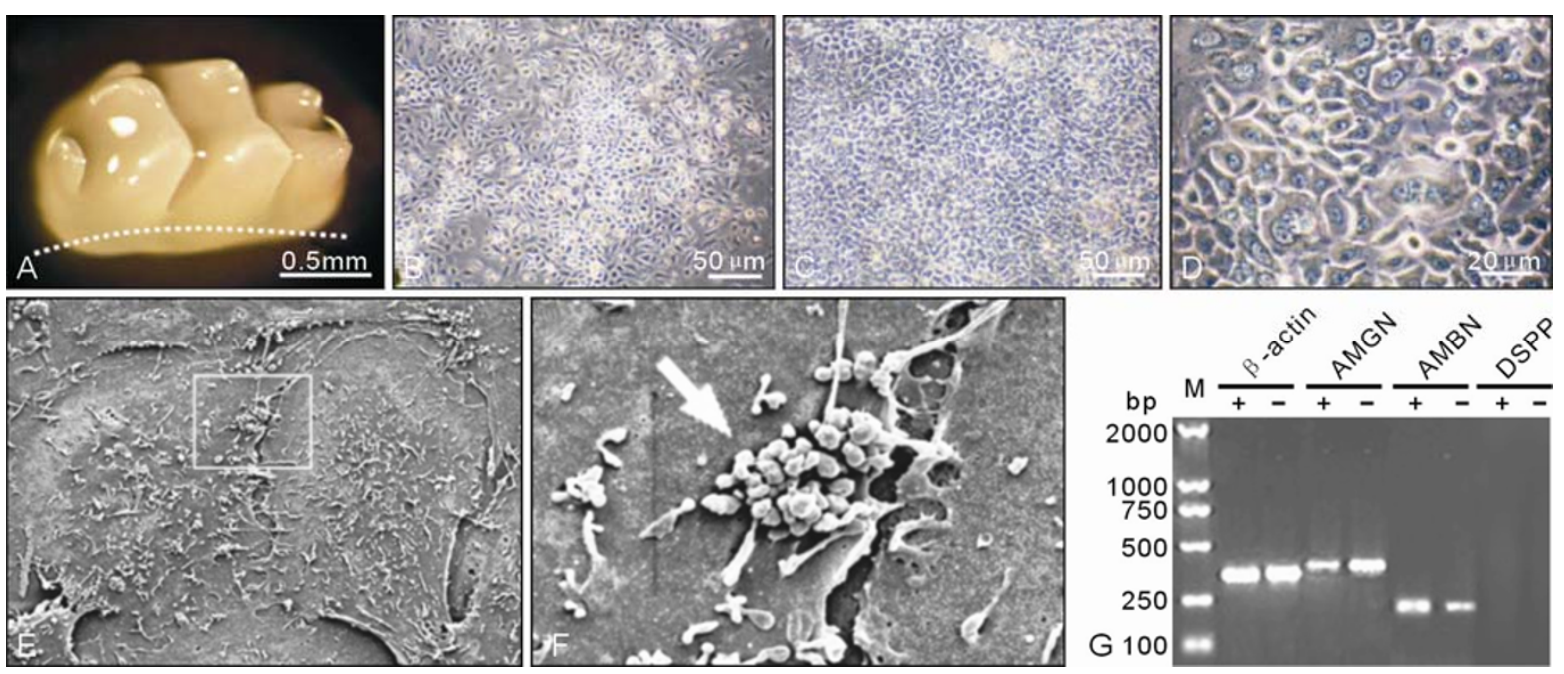

Figure 3 Conditioned medium from apical portion of molar germs (APMG-CM) promotes the differentiation of ABCs

(A): Macroscopic view indicated by dotted line showing APMG was dissected from the border of crown and root of 5-dpn SD rat first madibular molar. (B): Cells isolated from the APMG contained a mixture of cells with both epithelial and fibroblast shape. (C): After induction by APMG-CM, ABCs exhibited more rapid proliferative response indicated by denser alignment. (D): In APMG-CM treated group nuclear mitosis was more frequently observed. (E, F): SEM analysis of ABCs in APMG-CM treated group. (F): Higher magnifications of the boxed area in (E). ABCs revealed numerous diffuse granules of extracellular matrix production after induction as indicated by white arrow. (G): RT-PCR analysis showed that the treated group had a lower level of amelogenin expression and higher level of ameloblastin expression.

ture, $\mathrm{ABCs}$ exhibited a more distinct proliferative response (Figure 3C). Notably, nuclear mitosis was more frequently seen in the treated group (Figure 3D). The scanning electron microscope (SEM) showed ABCs featured characteristic cuboidal and highly flattened morphology (Figure 3E). Furthermore, adjacent cells revealed intimate intercellular contacts with microvilli structures. In higher magnifications, $\mathrm{ABCs}$ revealed numerous diffuse granules of extracellular matrix production in the treated group (Figure 3F). The overall morphological changes implied a more specialized function which means more differentiated state of $\mathrm{ABCs}$ treated with DAC-CM. To confirm this suggestion, RT-PCR was performed to investigate the expression of two differentiation-associated markers, amelogenin and ameloblastin in both groups (Figure 3G). Normalized against $\beta$-actin, semi-quantitative RT-PCR showed that the treated group had a lower level of amelogenin expression and higher level of ameloblastin expression. No DSPP mRNA expression was observed in either groups. With ameloblastin appearing mainly in differentiated ameloblasts, these findings indicate 
that DAC-CM could promote DECs to differentiate along its committed lineage.

\section{Discussion}

Previous study proved that as a specialized epithelial compartment at the apical end of rodent incisors, the apical bud is a stem cell-enriched structure comprising a large amount of the stellate reticulum, from which stem cells migrate to the inner enamel epithelium and contribute to a pool of proliferating cells, also known as transitamplifying cells. In sharp contrast with the in situ identification and characterization of apical bud structure, the in vitro analysis of cells from such unique structure has not come to the fore. We have demonstrated that the inductive microenvironment generated by ABCs could promote the lineage-specific differentiation of undifferentiated dental pulp stem cells and even skew the differentiation of non-odontogenic cells such as bone marrow stromal stem cells and follicle dermal papilla mesenchymal cells towards odontogenic cells (Yu et al., 2007). When ABCs were combined with dental pulp stem cells distinct morphogenesis can be observed within the combinants retrieved from ectopic renal capsule (Yu et al., 2007). From these previous findings we reasonably suppose that dental adult epithelial stem cells could exist within the apical portion of continuously growing teeth.

In the present study, we first focused on characterization of ABCs in terms of their culture morphology and biological phynotype. Although cells in the primary culture showed the heterogeneous feature to some extent, only epithelial-like cells existed in the subsequent culture under defined conditions. Furthermore, in our culture system ABCs exhibited typical phenotype traits reminiscent of those markers expressed in vivo. Cultured $\mathrm{ABCs}$ shared considerable homology with their in situ counterparts as to positive expression of CK14 and AMGN. Exclusion of dental papilla cells was corroborated by negative expression of mesenchymal marker vimentin. Occurrence of ALP mRNA detected by RT-PCR indicated the spontaneous mineralization ability of $\mathrm{ABCs}$ during culture. These results are in agreement with the previous reports which demonstrate that dental epithelial stem cells reside in the apical bud of mouse lower incisors and these cells produce IEE cells as transitamplifying progenitor cells which differentiate into ameloblasts (Harada et al., 1999; Kawano et al., 2004; Harada et al., 2004). In circumstances where adult stem cells survive and proliferate, they undergo the course during which the asymmetric cell division results in the production of one daughter cell remaining in the stem cell compartment and another undergoing further cell divisions and giving rise to differentiated cells (Harada et al., 1999; Morrison et al., 1997). The lineage-specific differentiation ability of cultured $\mathrm{ABCs}$ suggests that the cells are a suitable in vitro assay system to analyze regulation of their differentiation. Importantly, when apical bud tissue is isolated from where it stays to develop and reimplanted into ectopic renal capsule, it can go on original developmental trait based on its potential odontogenic capability to form crown-like tissue. These results clearly distinguished ABCs from cells in HERS with respect to their phenotype and differentiation ability.

Almost all the related findings revealed apical bud of murine incisors as a specific structure composed of a large amount of the stellate reticulum and the basal epithelium, which morphologically belongs to the tooth crown structure. Thus the fact that such a crown-like structure exists in a putative root position aroused our interest. With the advanced crown development, there are two developmentally determined directions which the cervical part of the crown has to obey. One is to adopt the "root" fate and another is still to being a "crown" (Tummers et al., 2003). Normally in mammalian molar, tooth roots develop to generate the final tooth shape with root and periodontium morphogenesis following tooth crown formation (Avery et al., 2001). During early root morphogenesis, the inner and outer enamel epithelia fuse to form a bilayered tissue called Hertwig's epithetlial root sheath (HERS). The HERS plays an important role during the initiation of mesenchymal cell proliferation and differentiation into odontoblasts during root development. Whether the real root/periodontal microenvironment could skew the biological performance of apical bud represents an unknown question. We have previously demons- 
trated that DAC possesses continuous developmental ability ectopically and may act as potent growth center of tooth root and periodontium, and can create a microenvironment to promote the differentiation and regenerative ability of periodontal ligament stem cells (Yang et al., 2008; Xu et al., 2008). By using the conditioned medium produced from DAC, we are destined to mimic the complex microenvironment produced by developing root and periodontium which are endowed with multiple signals essential for root/ periodontal complex development. Under the influence of DAC-CM, ABCs presented an accelerated differentiation process as indicated by observation of some transit-amplifying cell related change as enhanced proliferative and secretory activity. Essentially, up-regulation of ameloblastin expression after DAC-CM induction implied that ABCs had undergone rapid differentiation through a functional phenotype toward a terminal state, which resembles the traits of HERS cells in their proliferation and differentiation feature. These findings reinforce the hypothesis that the signaling milieu from a developing tooth root can dampen routine crown-like structure formation by accelerating differentiation of the dental epithelial cell. The murine molar represents an example where the entire cervical loop switches to root, whereas the murine incisor maintains the capacity in most of its cervical loop to produce crown. The enhanced marker for a differentiation state may imply that the cells from crown-like apical bud opt to take a crown-to-root transition under specific extrinsic cues. Harada et al. demonstrated that Fgf10 is the key determinants of choice for crown and root development (Yokohama-Tamaki et al., 2006). From the present results it is suggested that different intracellular and transcriptional factors respectively control the tooth crown and root development which can be influenced by the developmental microenvironment.

\section{Conlusion}

Taken together, in the present study we successfully isolated and characterized the ABCs as dental epithelial stem cells capable of ameloblastic differentiation. Furthermore, the results were conclusive in demonstrating that the normal traits of ABCs can be disrupted and biased by DAC microenvironment. Equally important the specific mechanisms required for the decisive factor in charge of ABCs and DAC microenvironment need further investigation and elucidation. This would improve our understanding of tooth development in terms of different signals participating in crown and root development.

\section{Acknowledgements}

This work was supported by National Nature Science Foundation of China (Project No.3057 2046, 30725042).

\section{References}

Avery JK (2001). Development of teeth: Root and supporting structures. In: Avery JK ed. Oral Development and Histology. Baltimore: Williams \& Wilkins, 13-28.

Hammarström L, Alatli I, Fong CD (1996). Origins of cementum. Oral Dis, 2(1): 63-69.

Harada H, Kettunen P, Jung HS, Mustonen T, Wang YA, Thesleff I (1999). Localization of putative stem cells in dental epithelium and their association with Notch and FGF signaling. $J$ Cell Biol, 147(1): 105-120.

Harada H, Ohshima H (2004). New perspectives on tooth development and the dental stem cell niche. Arch Histol Cytol, 67(1): 1-11.

Harada H, Toyono T, Toyoshima K, Yamasaki M, Itoh N, Kato S, et al. (2002). FGF10 maintains stem cell compartment in developing mouse incisors. Development, 129(6): 1533-1541.

Jernvall J, Thesleff I (2000). Reiterative signaling and patterning during mammalian tooth morphogenesis. Mech Dev, 92(1): 19-29.

Jinga VV, Gafencu A, Antohe F, Constantinescu E, Heltianu C, Raicu M, et al. (2000). Establishment of a pure vascular endothelial cell line from human placenta. Placenta, 21(4): 325-336.

Kawano S, Saito M, Handa K, Morotomi T, Toyono T, Seta $\mathrm{Y}$, et al. (2004). Characterization of dental epithelial progenitor cells derived from cervical-loop epithelium in a rat lower incisor. J Dent Res, 83(2): 129133.

Luan X, Ito Y, Diekwisch TG (2006). Evolution and development of Hertwig's epithelial root sheath. Dev 
Dyn, 235(5): 1167-1180.

Morotomi T, Kawano S, Toyono T, Kitamura C, Terashita M, Uchida T, et al. (2005). In vitro differentiation of dental epithelial progenitor cells through epithelialmesenchymal interactions. Arch Oral Biol, 50(8): 695705.

Morrison SJ, Shah NM, Anderson DJ (1997). Regulatory mechanisms in stem cell biology. Cell, 88(3): 287298.

Ohshima H, Nakasone N, Hashimoto E, Sakai H, Nakakura-Ohshima K, Harada H (2005). The eternal tooth germ is formed at the apical end of continuously growing teeth. Arch Oral Biol, 50(2): 153-157.

Pispa J, Thesleff I (2003). Mechanisms of ectodermal organogenesis. Dev Biol, 262(2): 195-205.

Smith CE, Warshawsky H (1975). Cellular renewal in the enamel organ and the odontoblast layer of the rat incisor as followed by radioautography using $3 \mathrm{H}-$ thymidine. Anat Rec, 183(4): 523-561.

Thesleff I, Vaahtokari A, Partanen AM (1995). Regulation of organogenesis. Common molecular mechanisms regulating the development of teeth and other organs. Int J Dev Biol, 39(1): 35-50.

Tucker A, Sharpe P (2004). The cutting-edge of mammalian development; how the embryo makes teeth. Nat Rev Genet, 5(7): 499-508.

Tummers M, Thesleff I (2003). Root or crown: a developmental choice orchestrated by the differential regulation of the epithelial stem cell niche in the tooth of two rodent species. Development, 130(6): 1049-1057.

Wise GE, Fan W (1989). Changes in the tartrate-resistant acid phosphatase cell population in dental follicles and bony crypts of rat molars during tooth eruption. J Dent Res, 68(2): 150-156.

Wu G, Deng Z, Fan X, Ma Z, Sun Y, Ma D, et al. (2008). Odontogenic Potential of Mesenchymal Cells from
Hair Follicle Dermal Papilla. Stem Cells Dev, Aug 1, [Epub ahead of print].

Xu L, Tang L, Jin F, Liu XH, Yu JH, Wu JJ, et al. (2009). The apical region of developing tooth root constitutes a complex and maintains the ability to generate root and periodontium-like tissues. $J$ Periodont Res, 44(2): 275-282.

Yang ZH, Zhang XJ, Dang NN, Ma ZF, Xu L, Wu JJ, et al. (2009). Apical tooth germ cell-conditioned medium enhances the differentiation of periodontal ligament stem cells into cementum/periodontal ligament-like tissues. J Periodontal Res, 44(2): 199-210.

Yokohama-Tamaki T, Ohshima H, Fujiwara N, Takada Y, Ichimori Y, Wakisaka S, et al. (2006). Cessation of Fgf10 signaling, resulting in a defective dental epithetlial stem cell compartment, leads to the transition from crown to root formation. Development, 133(7): 13591366.

Yu J, Deng Z, Shi J, Zhai H, Nie X, Zhuang H, et al. (2006). Differentiation of dental pulp stem cells into regular-shaped dentin-pulp complex induced by tooth germ cell conditioned medium. Tissue Eng, 12(11): 3097-3105.

Yu J, Wang Y, Deng Z, Tang L, Li Y, Shi J, et al. (2007). Odontogenic capability: bone marrow stromal stem cells versus dental pulp stem cells. Biol Cell, 99(8): 465-474.

Yu JH, Shi JN, Deng ZH, Zhuang H, Nie X, Wang RN, et al. (2006). Cell pellets from dental papillae can reexhibit dental morphogenesis and dentinogenesis. Biochem Biophys Res Commun, 346(1): 116-124.

Zeichner-David M, Oishi K, Su Z, Zakartchenko V, Chen LS, Arzate H, et al. (2003). Role of Hertwig's epithelial root sheath cells in tooth root development. Dev Dyn, 228(4): 651-63.

\footnotetext{
${ }^{\text {a }}$ Authors who contributed equally to this article.

*Corresponding authors:

Yan Jin

Adress: Department of Oral Histology \& Pathology, School of Stomatology, Research and Development Center for Tissue Engineering, Fourth Military Medical University, 145 West Hangle Road, Xi'an 710032, China

Tel: $862984776147 \quad$ Fax: $862983218039 \quad$ E-mail: yanjin@fmmu.edu.cn

Ling-ying Wen

Adress: Department of Paediatric Dentistry, School of Stomatology, Fourth Military Medical University, 145 West Hangle Road, Xi'an 710032, China
}

Tel: $862984776239 \quad$ E-mail: peosh@fmmu.edu.cn 